$6 / 46(13 \%), p=0.423]$. In the negative conversion group, the number of patients diagnosed with SLE was significantly higher than the control group [SLE, 3/14 $(21 \%)$ vs. $1 / 46(2 \%), p=0.036]$. However, thrombosis had recurred in one out of three patients with SLE.

Conclusions: The cumulative incidence of thrombotic events was not significantly different between negative conversion group and control group.

Disclosure of Interest: None declared

DOI: 10.1136/annrheumdis-2017-eular.5787

\section{SAT0308 CLINICAL, BIOLOGICAL AND IMMUNOLOGICAL FEATURES OF SJÖGREN SYNDROME: A STUDY OF 270 TUNISIAN PATIENTS}

T. Ben Salem, I. Naceur, M. Lamloum, I. Ben Ghorbel, M.H. Houman. Internal Medicine, Rabta university hospital, Tunis, Tunisia

Background: Sjögren syndrome (SS) is a chronic autoimmune disease characterized by a sicca syndrome and a wild spectrum of extra-glandular manifestations. Objectives: The aim of this study was to describe clinical, biological and immunological characteristics of patients with SS and to compare them in primary and associated SS.

Methods: We conducted a monocentric, retrospective study over a period of 15 years. Patients who fulfilled the American European Consensus Group criteria for Sjögren syndrome were enrolled.

Results: SS was diagnosed in 270 patients. The sex-ratio female/male was 10.73. The mean age at disease onset was 45 years $+/-13$ years (range 15-74 years) and at diagnosis was 47 years $+/-13$ years (range 15-76) with a mean delay of 3 years (range $0-25$ years). Sicca syndrome revealed the disease in most cases; both ocular and buccal dryness $(n=48)$, xerophtalmia $(n=21)$, xerostomia $(n=21)$ or parotid gland swelling $(n=17)$. SS was also revealed by joint involvement $(n=39)$, neurological manifestations $(n=26)$ or interstitial lung disease $(n=14)$. SS was systematically screened in 27 patients with another autoimmune disease and was found in 3 mothers who had a child with congenital atrio-ventricular bloc. Patients complained of xerophtalmia and xerostomia in respectively $95.2 \%$ and $95 \%$ of cases. Minor salivary biopsy was positive in $92.6 \%$ of cases. Parotid gland swelling was noted in 40 cases. Arthralgia and arthritis were respectively noted $77 \%$ and $18 \%$ of cases whereas myalgia and myositis were found in $11 \%$ and $5 \%$ of patients. Patients had pulmonary involvements in $27 \%$ of cases. Peripheral and central nervous system involvements were confirmed in $22 \%$ and $13 \%$ of cases and 12 patients presented with psychiatric disorders. Raynaud's phenomenon and purpura were noted in 48 and 17 patients. Biological data showed lymphopenia $(n=115)$, anemia $(n=83)$, thrombocytopenia $(n=25)$ and hypergammaglobulinemia $(n=151)$. Antinuclear antibodies were positive in 210 cases; anti-SSA and antiSSB were present in respectively $57 \%$ and $37 \%$ of patients. SS was primary in 155 patients and was associated to another autoimmune disease in 113 patients; systemic lupus erythematosus $(n=48)$, rheumatoid arthritis $(n=20)$, systemic sclerosis $(n=19)$, autoimmune liver disease $(n=13)$ and auto-immune thyroiditis $(n=9)$. Arthralgia ( $91.2 \%$ vs $66.2 ; p<0.0001)$, arthritis ( $35.7 \%$ vs $5.2 \% ; p<0.0001)$, myalgia ( $17 \%$ vs $7.1 \% ; p=0.012$ ) and Raynaud's phenomenon ( $33.3 \%$ vs $7.7 \%$; $\mathrm{p} \leq 0.0001)$ were significantly less frequent in primary SS. Lymphopenia $(61.1 \%$ vs $32.9 \% ; p<0.0001)$, anemia (48.6\% vs $19.5 \% ; p<0.0001)$, inflammatory syndrome ( $35.8 \%$ vs $17.9 ; p=0.002)$ and ANA ( $92 \%$ vs $70.9 \% ; p<0.0001)$ were significantly more frequent in associated SS. Corticosteroids and immunosuppressive therapy were used in respectively 141 and 90 patients because of severe complications. Only 3 case of lymphoma were observed and 3 patients died. The mean duration of follow up was 50 months.

Conclusions: Sicca syndrome is the major symptom of SS but extra-glandular manifestations are less frequent and can be serious causing disability specially neurologic and pulmonary involvements. Some manifestations are significantly more frequent in patients with associated autoimmune diseases like joint involvements, Raynaud's phenomenon, anemia and lymphopenia. These manifestations are mainly related to SLE, rheumatoid arthritis and systemic sclerosis.

Disclosure of Interest: None declared

DOI: 10.1136/annrheumdis-2017-eular.3589

\section{SAT0309 CAN WE FORESEE SLE IN ITP PATIENTS? TO DISTINGUISH ITP PATIENTS WITH HIGH RISK OF SLE BY A NATIONWIDE COHORT STUDY-BASED DECISION TREE}

T.-H. Li ${ }^{1}$, Y.-S. Chang ${ }^{2}$, C.-Y. Tsai ${ }^{3} .{ }^{1}$ Division of Allergy, Immunology and Rheumatology, Department of Internal Medicine, Chiayi Branch, Taichung Veteran General Hospital, Chiayi City; ${ }^{2}$ Division of Allergy, Immunology and Rheumatology, Department of Internal Medicine, Taipei Hospital, Ministry of Health and Welfare, New Taipei City; ${ }^{3}$ Division of Allergy, Immunology and Rheumatology, Department of Internal Medicine, Taipei Veteran General Hospital, Taipei City, Taiwan, Province of China

Background: Immune thrombocytopenic purpura (ITP) is an autoimmuneassociated thrombocytopenia which is occasionally the initial presentation of systemic lupus erythematosus (SLE), and thus periodical following up has been suggested. Whereas long-term surveillance on all ITP patients would be time and cost-consuming, and thus to distinguish those with high probability of SLE development among ITP patients should be more practical.
Objectives: To distinguish ITP patients with high risk of SLE development by a decision tree model.

Methods: We enrolled ITP patients without previous SLE diagnosis from the National Health Insurance research database between 1997 and 2012 and identified those certificated with catastrophic illness of SLE during follow up, by which the diagnosis was reconfirmed by another rheumatologists. We also analyzed the symptoms and comorbidities as well as the dose of average oral steroid to derive the decision trees, which classified the ITP patients with different probability of development of SLE.

Results: A total of 10,265 ITP patients were enrolled, among whom 80 patients developed SLE while following-up. The whole ITP patients were allocated to training group (7,186 patients including 57 with SLE) and testing group (3,079 patients including 23 with SLE); the former was used for derivation of the decision-tree based model and the latter for validation of the previously mentioned model, and provided high sensitivity $(78.2 \%)$, specificity (99.2\%) and negative prediction value $(99.8 \%$, Fig.). To reduce the complexity, we also pruned our decision tree to propose less-complicated models.

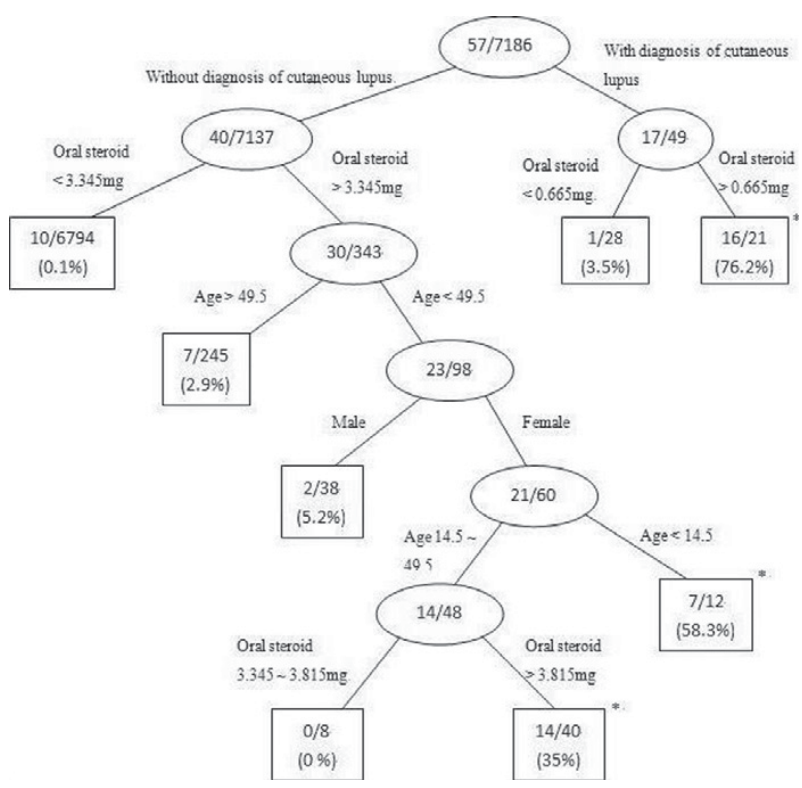

Conclusions: We derived classification decision tree suitable for various clinical scenarios of ITP patients, among whom those with high probability of development of SLE would be distinguished.

References:

[1] Balsalobre Aznar J, Herráez Herrera P, Porta Etessam J, Torres Martín C, Bermell Serrano JC, Núñez López R, et al. Idiopathic thrombocytopenic purpura as first manifestation of systemic lupus erythematosus lupus. An Med Interna. 1999:16:611-4.

[2] Risk factors for future development of systemic lupus erythematosus in children with idiopathic thrombocytopenic purpura. Pediatr Blood Cancer. 2006;47(5 Suppl):657-9.

Disclosure of Interest: None declared

DOI: 10.1136/annrheumdis-2017-eular.1359

\section{SAT0310 ULTRASONOGRAPHIC SCORING OF THE MAJOR SALIVARY GLANDS IN SJOGREN'S SYNDROME: A COMPARATIVE STUDY WITH DISEASE ACTIVITY INDEXES}

N. İnanç ${ }^{1}$, Y. Yalçınkaya ${ }^{1}$, G. Mumcu $^{2}$, Z. Ertürk ${ }^{1}$, A.U. Unal ${ }^{1}$, P. Atagündüz ${ }^{1}$ H. Direskeneli ${ }^{1} .{ }^{1}$ Department of Internal Medicine, Division of Rheumatology, Marmara University, School of Medicine; ${ }^{2}$ Marmara University, Faculty of Dentistry, Istanbul, Turkey

Background: Sjogren's syndrome (SjS) is characterised by chronic autoimmune inflammation primarily affects the salivary and lacrimal glands. Recently, ultrasonography (USG) of major salivary glands (SG-USG) has been used to evaluate salivary glands in primary and secondary SjS.

Objectives: We aimed to investigate the association between the ultrasonographic scoring of major salivary glands and disease activity indexes in patientswith primary SjS.

Methods: Forty-two primary SjS patients fulfilling ACR-EULAR classification criteria (2002) were included. Disease activity indexes (Sjögren's Syndrome Patients Reported Index (ESSPRI), Visual Analogue Scale (VAS), EULAR Sjögren's Syndrome Disease Activity Index (ESSDAI)] were recorded. Major salivary glands (bilateral parotis and submandibular glands) were scored according to two different scoring system [Hocevar A. (0-48) ve Milic VD. (0-12)].

Results: Demographics, clinical characteristics, disease activity indexes and SG-USG scores were summarised in table 1 and table 2. 